Document downloaded from:

http://hdl.handle.net/10251/38236

This paper must be cited as:

Garcia Panach, J.; Lull Noguera, N.; Lull Noguera, JJ.; Ferri Domínguez, J.; Martínez, C.; Sopena, P.; Robles Viejo, M.... (2011). A Voxel-based analysis of FDG-PET in traumatic brain injury: regional metabolism and relationship between the thalamus and cortical areas. Journal of Neurotrauma. 28(9):1707-1717. doi:10.1089/neu.2011.1851.

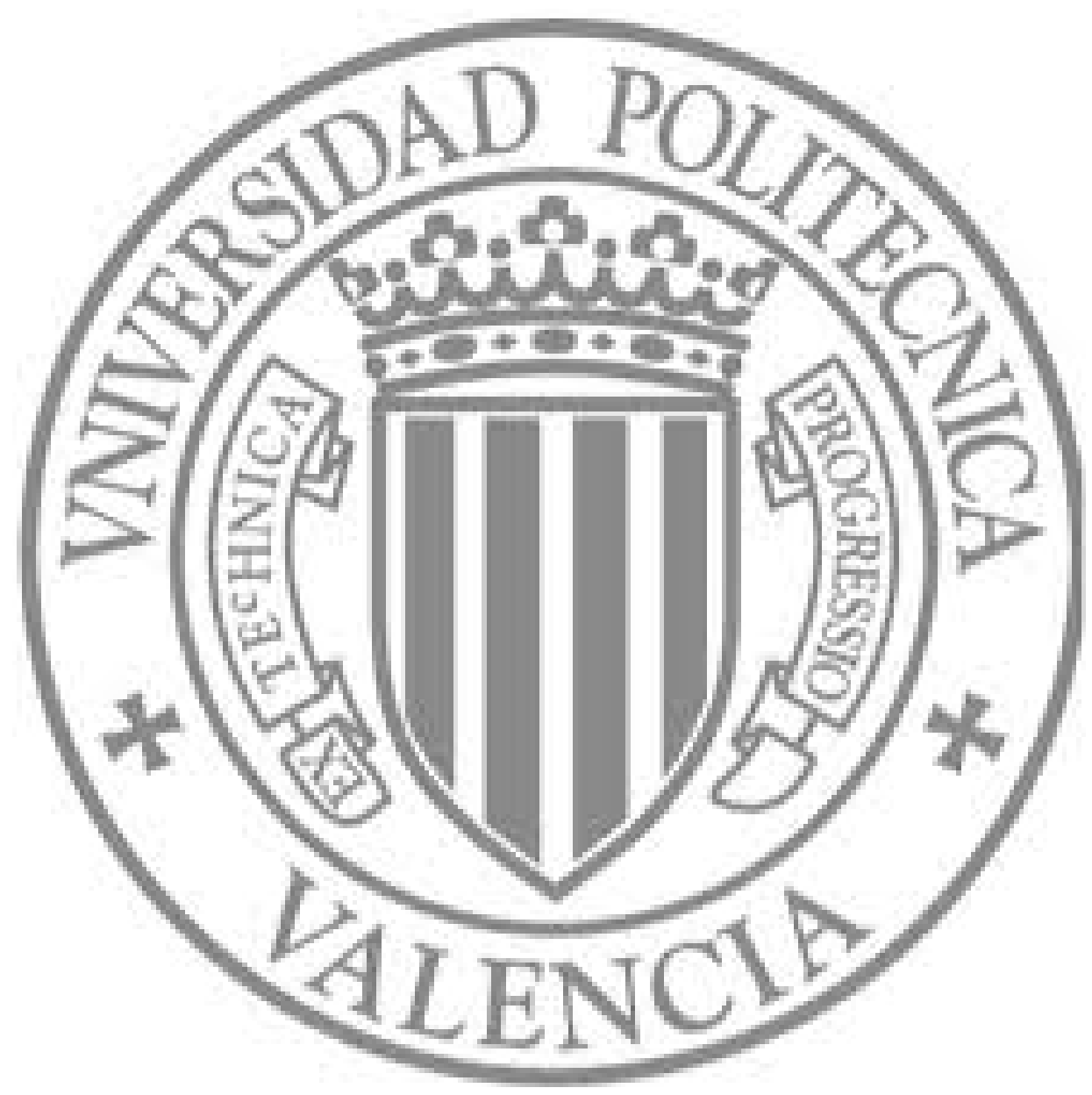

The final publication is available at

hppt://dx.doi.org/10.1089/neu.2011.1851

Copyright Mary Ann Liebert 


\section{A Voxel-based analysis of FDG-PET in traumatic brain injury: regional metabolism and relationship between the thalamus and cortical areas \\ Javier García-Panach ${ }^{1,2}$, Nuria Lull ${ }^{1,3}$, Juan José Lull ${ }^{1}$, Joan Ferri ${ }^{2}$, Carlos Martínez ${ }^{4}$, Pablo Sopena ${ }^{4}$, Montserrat Robles ${ }^{1}$, Javier Chirivella ${ }^{2}$, Enrique Noé ${ }^{2}$ \\ ${ }^{1}$ Grupo de Informática Biomédica, Instituto de Aplicaciones de las Tecnologías de la Información y de las Comunicaciones Avanzadas (ITACA), Universidad Politécnica de Valencia. Valencia, Spain \\ ${ }^{2}$ Servicio de Daño Cerebral de los Hospitales NISA. Fundación Instituto Valenciano de Neurorrehabilitación (FIVAN). Valencia, Spain \\ ${ }^{3}$ Universidad CEU Cardenal Herrera. Valencia, Spain \\ ${ }^{4}$ Servicio PET-Medicina Nuclear, Hospital 9 de Octubre, Valencia, Spain}

Address for correspondence:

Dr. Enrique Noé

Servicio de Neuro-Rehabilitación

Hospital NISA Valencia al Mar

C/ Río Tajo nº 1

46011 Valencia (ESPAÑA)

Phone: (34) 963.352.500; Fax: (34) 963.352.501; e-mail: enoe@comv.es

Keywords: Consciousness. PET, Functional Imaging. Cerebral Metabolism. Head Injury. Image Analysis.

Word count: Abstract (250) / Paper (3490) 


\section{ABSTRACT}

Objective: To study the correlations and the differences in glucose metabolism between the thalamus and cortical structures in a sample of severe traumatic brain injury (TBI) patients with different neurological outcomes. Methods: We studied 49 patients who had suffered a severe TBI and 10 healthy control subjects using 18F-FDG-PET. The patients were divided into three groups: the MCS\&VS group $(n=17)$, which included patients who were in a vegetative or a minimally conscious state; the In-PTA group $(\mathrm{n}=12)$, which included patients in post-traumatic amnesia (PTA); and the Out-PTA group $(n=20)$, which included patients who had recovered from PTA. SPM5 software was used to determine the metabolic differences between the groups. FDG-PET images were normalized and four regions of interest were generated around the thalamus, precuneus and the frontal and temporal lobes. The groups were parameterized using the Student's T-test. Principal component analysis was used to obtain an intensityestimated-value per subject to correlate the function between the structures. Results: Differences in glucose metabolism in all structures were related to the neurological outcome, and the most severe patients showed the most severe hypometabolism. We also found a significant correlation between the cortico-thalamocortical metabolism in all groups. Conclusions: Voxel-based analysis suggests a functional correlation between these four areas and decreased metabolism was associated with less favorable outcome. Higher levels of activation of the corticocortical connections appear to be related to better neurological conditions. Differences in the thalamo-cortical correlations between patients and controls may be related to traumatic dysfunction due to focal or diffuse lesions. 


\section{INTRODUCTION}

The clinical consequences of traumatic brain injury (TBI) are directly related to factors derived from the actual trauma, such as the severity, mechanism, location or duration of the impact.[1] It is now commonly accepted that the recovery of motor, cognitive, behavioral and emotional deficits after a TBI requires a multidisciplinary and individualized therapeutic approach that is based on the information provided by the factors mentioned above.[2] Therefore, neuroimaging techniques can help both in the understanding of the neuroanatomical basis of these changes and in the understanding of the diversity and heterogeneity of the clinical course of these patients. In addition, neuroimaging data could be used in the near future to guide the most effective therapeutic approaches in patients with TBI.

Typically, moderate/severe TBI causes abrupt transfer of mechanical energy into the brain parenchyma, which results in injuries that disrupt normal neurological function. This mechanical energy can result in contusive focal lesions that usually appear in the cortical structures and diffuse axonal injury (DAI) that usually follows a depth gradient that is dependent on the intensity of the forces exerted at the cephalic level.[3] The importance of DAI has been recently highlighted when it was demonstrated that these diffuse lesions play an important role in the cognitive impairment and decreased awareness after TBI. However, subtle diffuse lesions may not appear on structural imaging, or if present, their extent or location are often not correlated with many of the clinical symptoms that are commonly observed in these patients. Therefore, there is increasing interest in developing and applying new functional neuroimaging techniques that are specifically designed for the detection of DAI.[4-5] 
The integrity of cerebral cortico-subcortical connectivity is essential for maintaining normal brain function.[6-7] This connectivity is particularly vulnerable to the effects of traumatic injuries that can alter not only the individual components of the network (focal lesions) but also the connections between brain-nodes (DAI). Because of its connections to the ascending reticular system and its role in cognitive information processing via its connections to the cerebral cortex, the thalamus seems play a central role in the network that is responsible for maintaining awareness.[8-10] Recently, Laureys et al. described a brain network that is involved in maintaining awareness after TBI and includes the thalamus, precuneus and the frontal cortex. [11] The functionality of brain network systems in TBI patients throughout the entire recovery process is currently being elucidated.

Our group has previously demonstrated that the thalamic hypometabolism that is detected by FDG-PET in patients who have suffered a severe TBI is directly related to the patients' neurological outcome.[12] Given the well-known thalamic connectivity to cortical regions, such as the precuneus or fronto-temporal cortex, which are especially vulnerable to the effects of a TBI, the study of the integrity of these systems is highly interesting. Characteristically, damage to these regions has been linked to deficits in complex neurocognitive functioning and behavioral changes that lead to a reduced functional recovery after a TBI.[13-14] In this paper, we extend our analysis to other structures of the cerebral cortex, including the frontal cortex, temporal cortex and precuneus. Our aim was to study the integrity of the connections between these cortical structures and the connections that are established between these cortical structures and deep brain structures such as the thalamus.

We used a variation of Voxel-Based Morphometry (VBM)[15-16], to analyze the data from metabolic FDG-PET images from a sample of patients with different 
neurological outcomes after TBI. This method, which was originally used for anatomical magnetic resonance (MR), has been previously used in the study of thalamic metabolism in TBI patients.[12] This method allows us to analyze, process and quantify the differences in FDG-PET signals with an independent treatment for each voxel of the image. Specifically, we have applied this method for the analysis of the regions of interest (ROI) involved in our study. Our hypothesis was that the metabolism of these structures and the functional networks that are established between them are related to the neurological and functional outcome after TBI. 


\section{MATERIAL AND METHODS}

\section{Subjects}

The sample included in this study has been previously described.[12] It consists of ten healthy controls (mean age 40.8 (SD 15.4) years; six men and four women) and 49 severe TBI patients, aged 16-65 years, who were divided into three clinical groups according to either their level of consciousness or their cognitive outcome. The classification of the groups was as follow: patients who were in a vegetative or minimally conscious state (MCS\&VS, $n=17$ ), patients who experienced post-traumatic amnesia (In-PTA, $n=12$ ) and patients who had recovered from PTA (Out-PTA, $n=20$ ). Demographic data from the TBI patients are shown in Table I. The classification criteria of this sample may also be reviewed in detail in our previous publication.[12]

The percentage of the patients who showed focal structural lesions on CT $(n=17)$ or MRI $(n=32)$ in the cerebral areas studied (thalamus, frontal cortex, temporal cortex and precuneus) was determined for each group (Table I). As expected, anatomical neuroimaging studies showed a low frequency of structural thalamic lesions and a higher frequency of structural cortical lesions, which were mainly located in the frontal cortex and temporal cortex. There was a trend towards significance $(\mathrm{p}=0.08)$ when the percentage of in-PTA patients with focal temporal lesion (75\%) was compared to that of the other groups. Due to the retrospective design of this study, data on lesions that specifically affected the precuneus were not available. As an indirect measure of the effects on the precuneus, the percentage of patients that showed parietal lesions were selected.

All patients provided written informed consent. For patients with a low level of consciousness, consent was obtained from a close relative. We also obtained approval 
for the study from the hospital ethics committee, and the study complied with the principles of the Declaration of Helsinki.

Table I. Comparison of characteristics between patients. Data are mean (SD)

\begin{tabular}{|c|c|c|c|c|}
\hline & $\begin{array}{c}\text { MCS\&VS } \\
(n=17)\end{array}$ & $\begin{array}{c}\text { In-PTA } \\
(n=12)\end{array}$ & $\begin{array}{c}\text { Out-PTA } \\
(\mathbf{n}=20)\end{array}$ & $\mathbf{P}$ \\
\hline Age (years) & $30.3(7.7)$ & $35.2(15.8)$ & $30.9(9)$ & NS \\
\hline Chronicity (days) & $293.9(234.3)$ & 255 (143.9) & $217(126)$ & NS \\
\hline Sex (male/female) & $14 / 3$ & $7 / 5$ & $17 / 3$ & NS \\
\hline Education (years) & $10(3.4)$ & $10(4)$ & $9.7(3.3)$ & NS \\
\hline Coma (days) & - & $42.9(58.6)$ & $19.9(18.9)$ & NS \\
\hline PTA (days) & - & - & $78.5(72.5)$ & - \\
\hline $\begin{array}{l}\text { Etiology }(\mathbf{n}) \\
\text { Traffic Motor/Pedestrian } \\
\text { Falls } \\
\text { Violent injuries }\end{array}$ & $\begin{array}{c}12 / 1 \\
1 \\
3\end{array}$ & $\begin{array}{c}6 / 1 \\
2 \\
3\end{array}$ & $\begin{array}{c}13 / 2 \\
3 \\
2\end{array}$ & NS \\
\hline $\begin{array}{l}\text { Thalamic lesion }-\mathbf{n}(\boldsymbol{\%}) \text { - } \\
\text { Absence } \\
\text { Presence }\end{array}$ & $\begin{array}{c}18(90.0 \%) \\
2(10.0 \%)\end{array}$ & $\begin{array}{c}11(91.7 \%) \\
1(8.3 \%)\end{array}$ & $\begin{array}{c}14(82.3 \%) \\
3(17.7 \%)\end{array}$ & NS \\
\hline $\begin{array}{l}\text { Frontal lesion } \mathbf{- n}(\boldsymbol{\%})- \\
\text { Absence } \\
\text { Presence }\end{array}$ & $\begin{array}{c}6(30.0 \%) \\
14(70.0 \%)\end{array}$ & $\begin{array}{l}6(50.0 \%) \\
6(50.0 \%)\end{array}$ & $\begin{array}{c}4(23.5 \%) \\
13(76.5 \%)\end{array}$ & NS \\
\hline $\begin{array}{l}\text { Temporal lesion }-\mathbf{n}(\%) \text { - } \\
\text { Absence } \\
\text { Presence }\end{array}$ & $\begin{array}{c}13(65.0 \%) \\
7(35.0 \%)\end{array}$ & $\begin{array}{l}3(25.0 \%) \\
9(75.0 \%)\end{array}$ & $\begin{array}{c}10(58.8 \%) \\
7(41.2 \%)\end{array}$ & 0.08 \\
\hline $\begin{array}{l}\text { Parietal lesion }-\mathbf{n}(\%)- \\
\text { Absence } \\
\text { Presence }\end{array}$ & $\begin{array}{c}13(65.0 \%) \\
7(35.0 \%)\end{array}$ & $\begin{array}{l}9(75.0 \%) \\
3(25.0 \%)\end{array}$ & $\begin{array}{c}11(64.7 \%) \\
6(35.3 \%)\end{array}$ & NS \\
\hline
\end{tabular}

\section{Acquisition of PET images}

All patients underwent a PET/CT brain scan after an intravenous injection of a 110-MBqkg-1 dose of 2-fluorine-2-deoxy-18F-D-glucose (FDG) up to a maximum dose of $370 \mathrm{MBq}$. The acquisition of the PET images was performed according to the methodology that was previously published by our group.[12] 


\section{Voxel-based PET Analysis}

We adapted the previously validated methodology[12], which is based on VoxelBased Analysis (VBA) of the images using MATLAB 7.4 software (The MathWorks, Natick, Massachusetts, USA) and SPM software (Statistical Parametric Mapping 5, Wellcome Department of Cognitive Neurology, Institute of Neurology, London, UK). Briefly, the PET images were subjected to the process of Voxel-Based Morphometry (VBM).[15] Image pre-processing consisted of the following steps: 1) image conversion to the Niftii format; 2) intensity normalization; 3) creation of the PET custom template; 4) spatial normalization; 5) smoothing; and 6) selection of the regions of interest (ROIs).

\section{Region of Interest Selection (ROI)}

The selection of each ROI was performed with the help of an expert neurologist. The ROIs selected for each mask were as follows: 1) Thalamus; 2) Precuneus; 3) Frontal cortex (composed of the orbitofrontal, dorsolateral and frontomedial areas); and

4) Temporal cortex (composed of the upper, middle and inferior temporal areas, the superior and middle temporal poles, the hippocampus, the parahippocampal gyrus and the fusiform gyrus).

\section{Metabolic differences between the groups of subjects.}

After obtaining the image with the selected ROI, a statistical analysis based on the general linear model (GLM software for SPM5) was conducted. A design matrix and a statistical test model for comparison were defined for each pair of groups in each structure: (controls compared to the MCS\&VS group, controls compared to the In-PTA group, controls compared to the Out-PTA group, the Out-PTA group compared to the MCS\&VS group, the Out-PTA group compared to the In-PTA group, and the In-PTA 
group compared to the MCS\&VS group as well as their opposites: the MCS\&VS group compared to the controls, and so on). The model fitting consisted of an estimation of the parameters to obtain the best approximation of the data to the model. An estimation of the model was carried out using the Student's two-sample t-test that was independently applied to each voxel based on contrast, with the aim of measuring the interactions and the possible differences between the groups. A different model was generated for each two-group comparison, and a technique that solved the multiple comparison problem (as thousands of voxels are univariately tested) and controlled the ratio of false positives (False Discovery Rate, FDR)[17] was used to establish the levels of statistical significance, with $\mathrm{p}<0.05$.

\section{Metabolism Correlation between structures.}

A one-sample-t-test was applied to each of the selected ROIs that allowed the principal components of each subject to be determined by the application of an eigenvariate function. The value of the first principal component of each structure in each subject was then extracted, the 2D correlation of the structures across subjects was performed. For each group of patients, the three cortico-cortical systems (frontotemporal, fronto-precuneus and temporo-precuneus) and the three cortico-subcortical systems (fronto-thalamic, temporo-thalamic and precuneo-thalamic) were correlated. Specifically, this study sought to correlate the metabolic intensity between the various brain structures in each subject. 


\section{RESULTS}

\section{Metabolic Differences between the Controls and the Study Patients}

We have previously reported on the global thalamic hypometabolism in TBI patients compared to controls.[12] Similar results were obtained when comparing the thalamic and frontal metabolism between the groups, and the most significant difference was found when comparing the controls to the MCS\&VS group. Significant differences were also found when comparing the controls to the In-PTA group, although lower than in the previous comparison. Finally, a less significant difference between the healthy controls and the patients was found in the comparison between the controls and the OutPTA group.

The precuneus and temporal cortex metabolic differences reached significance both in the comparison between the controls and the MCS\&VS group and between the controls and the In-PTA group, with greater differences in the first comparison. However, in these structures, no significant metabolic differences were obtained when comparing the controls to the Out-PTA group.

No voxel survived the reverse tests (the MCS\&VS group compared to controls; the In-PTA group compared to the controls; and the Out-PTA group compared to the controls) in the four brain structures. These results are shown in Figure 1.

\section{Insert figure 1 about here}

\section{Metabolic Differences between Patients}

We found significant thalamic metabolic differences in the comparisons between the patients in the three groups. The comparison between the Out-PTA group and the MCS\&VS group showed the largest metabolic differences between of all of the patient

groups. In addition, significant thalamic metabolic differences appeared when 
comparing the Out-PTA group to the In-PTA group. The smallest metabolic differences were found in the thalamus, in the comparison between the In-PTA group and the MCS\&VS group although these differences reached statistical significance.

The frontal and temporal cortices showed significant metabolic differences in the comparisons between the Out-PTA and the MCS\&VS groups and the Out-PTA and the In-PTA groups with a greater significance of the Out-PTA and MCS\&VS comparison, however no voxel survived the comparison between the In-PTA group and the MCS\&VS group.

Significant metabolic differences were found in the precuneus only when comparing the Out-PTA group to the MCS\&VS group. No significant differences were found in the other two comparisons (between the Out-PTA group and the In-PTA group and between the In-PTA group and the MCS\&VS group).

No voxel survived the reverse tests (the MCS\&VS group compared to the Out-PTA group; the In-PTA group compared to the Out-PTA group; and the MCS\&VS group compared to the In-PTA group) in the four brain structures. These results are shown in Figure 2.

\section{Insert figure 2 about here}

Results are presented in the form of parametric probability maps where the intensity or brightness of each voxel was determined by its corresponding z statistic (Table II).

Table II. Representation of studies and significant values: maximum Z-values (metabolism intensity), Ke (number of voxels).

\begin{tabular}{|c|c|c|c|c|c|c|c|c|}
\hline & \multicolumn{2}{|c|}{ Thalamus } & \multicolumn{2}{|c|}{ Frontal } & \multicolumn{2}{|c|}{ Temporal } & \multicolumn{2}{|c|}{ Precuneus } \\
\hline & Z Value & $\mathrm{Ke}$ & Z Value & $\mathrm{Ke}$ & Z Value & $\mathrm{Ke}$ & Z Value & $\mathrm{Ke}$ \\
\hline Controls $>$ MCS\&VS & 7,07 & 4260 & 6,39 & 21709 & 5,44 & 24382 & 5,18 & 3857 \\
\hline Controls > In-PTA & 5,88 & 3758 & 5,62 & 20700 & 4,42 & 23189 & 3,91 & 1939 \\
\hline Controls > Out-PTA & 3,87 & 1942 & 4,15 & 3957 & - & - & - & - \\
\hline Out-PTA > MCS\&VS & 6,07 & 3533 & 5,97 & 20884 & 4,73 & 18576 & 5,21 & 2922 \\
\hline Out-PTA > In-PTA & 4,15 & 1664 & 4,42 & 8534 & 4,61 & 8830 & - & - \\
\hline In-PTA > MCS\&VS & 3,60 & 1140 & - & - & - & - & - & - \\
\hline
\end{tabular}




\section{Analysis of the Correlation between Structures}

There was a strong correlation between the cortical structures in both patients and controls. However, we obtained a weaker correlation in the control group when comparing the cortical structures to the thalamus and particularly when analyzing the correlation between the thalamus and the frontal cortex. The quantitative results obtained in this study are showed in Table III.

Table III. Correlation between structures in the four groups of subjects.

\begin{tabular}{llcccc}
\hline & & Controls & Out-PTA & In-PTA & MCS\&VS \\
\hline Thalamus & Frontal & 0.32 & 0.72 & 0.76 & 0.64 \\
Thalamus & Temporal & 0.59 & 0.76 & 0.77 & 0.82 \\
Thalamus & Precuneus & 0.50 & 0.80 & 0.92 & 0.71 \\
Frontal & Temporal & 0.86 & 0.82 & 0.70 & 0.85 \\
Frontal & Precuneus & 0.88 & 0.80 & 0.89 & 0.88 \\
Temporal & Precuneus & 0.88 & 0.85 & 0.81 & 0.87 \\
\hline
\end{tabular}

The chart in Figure 3 shows the intensity data in each structure for each subject; the basis for the correlation analysis was divided into four parts, one for each group of subjects (Controls, Out-PTA, In-PTA, MCS\&VS). Each line shows the current value of each structure (thalamus, precuneus, frontal cortex, temporal cortex) - (Figure 3). 


\section{DISCUSSION}

Our results show that the hypometabolism that was detected not only in widespread cortical areas (fronto-temporal cortex and precuneus) but also in the thalamus after TBI typically resolves as long as the patient's neurological outcome improves. The strong correlations between the metabolism in each of our patients' brain structures, reinforces the idea that functional neural networks connect close and distant structures, which is the basis for the normal brain function.[18] These results are consistent with those of other groups that describe the existence of a significant correlation between the severity of the TBI and global cerebral hypometabolism.[19-23] Using more sensitive image analysis techniques, our group previously demonstrated the metabolic differences in specific brain structures when comparing severe TBI patients in different clinical situations.[12] In particular, the previously described findings related to thalamic metabolism have been extended to other cortical regions in this study.

Voxel-Based Morphometry studies conducted in patients in a vegetative state have described an impairment of a broad network that covers the thalamic nuclei and the polymodal associative cortices (encompassing the bilateral prefrontal regions, Broca's area, the parieto-temporal and posterior parietal areas, and the precuneus cortex).[11, 24-25] These studies have also shown that clinical improvement of these states is associated with a functional restoration of the connections between the intralaminar thalamic nuclei and the prefrontal cortex. $[8,26]$ Our analysis of the metabolic changes by regions of interest demonstrated that the thalamus seems to be the most sensitive structure that shows differences when comparing any neurological state.[12] As expected, due to the characteristics of our sample, the intergroup comparative study showed that the largest metabolic differences appeared when comparing healthy controls to the patients in a vegetative and minimally conscious state. To date, three 
possible explanations for this thalamic hypometabolism have been proposed; direct focal lesions, damage to the white matter resulting from diffuse axonal injury or a phenomenon of remote disconnection / deafferentation.[8, 11, 22, 26-27] Recent studies have focused on thalamic metabolism in TBI patients, including our previous results[12], and they support the contribution of the latter two listed mechanisms due to the functional or anatomical disconnection between the cerebral cortex and the deeper brain regions.[11, 22, 28-29] Metabolic data from other brain structures that were analyzed in this study supports deafferentation as a basic mechanism that may explain the thalamic hypometabolism detected in our patients.

According to our results, the recovery of the metabolism in the frontal cortex seems to represent the most sensitive cortical marker in the detection of clinically relevant changes in our sample of patients. To date, several studies have demonstrated the importance of preserving frontal metabolism in patients who have suffered a TBI.[1314] Damage to the frontal lobe and its connections are particularly detrimental and compromise long-term outcome by interfering with the restoration of normal personality, occupational function and community reintegration. Given the recognized importance of the fronto-thalamic connectivity, it was not surprising that the hypometabolism detected in this system appeared to be the most relevant dysfunction of our sample, with the expected resultant clinical implications.[22, 26]

Unlike thalamic metabolism, fronto-temporal metabolism showed no significant differences when comparing patients in PTA with those in VS and MCS. The major metabolic differences in these two cortical areas began to appear in the comparisons between patients who were in PTA compared to those who had already recovered from this state. These data suggest that the recovery from PTA could be associated with an overall improvement in cerebral cortical function. To our knowledge, functional 
neuroimaging studies that compare patients during and after the PTA period are still not available. Previous structural neuroimaging studies in PTA patients have shown a preferential involvement of the medial temporal cortex, subfrontal area, and the central brain structures or the connections between both.[30-31] According to our results, structural neuroimaging data showed a modest tendency for more temporal lesions in PTA patients compared to other groups, but the reason for the relationship between the duration of PTA and a more widespread brain dysfunction remains unknown. Our data of the fronto-temporal metabolism recovery after PTA resolution should be cautiously interpreted because our results are based on cross-sectional comparisons. Future longitudinal studies that include patients before and after PTA resolution would help to elucidate this question.

Our results comparing the regional cortical metabolism with the inclusion of healthy controls can help us understand the role of these areas in the neurological recovery after TBI. In the case of the temporal cortex, there were no significant differences between the healthy controls and the patients who had recovered from PTA, which suggests that the activation of the temporal cortex seems to play an important role in the earlier stages, especially during PTA resolution when memory processes related to recent events begin to operate.[32] In the case of the frontal cortex, the differences found between the healthy controls and the patients who had recovered from PTA again highlight the importance of the normal functioning of the frontal cortex in the final outcome after TBI.[13-14] The different evolution of the metabolism of these two cortical structures could also be due to differences in the distribution of lesions detected by structural imaging or to the classification criteria used in this study. It is possible that a classification based on more specific criteria, which are directly related to the functionality of these areas, would have yielded different results. It is also 
possible that the changes found in frontal metabolism compared to other cortical structures could be explained by the effect of diffuse lesions. There is still no clear explanation to demonstrate the particular vulnerability of frontal metabolism to the effects of DAI lesions. It has been suggested that the strong convergence of white matter fibers in the frontal lobes may explain why lesions in any region of the brain tend to show a proportionally robust effect in this broad associative cortical area.[33]

The precuneus seems to be involved in modulating consciousness via corticosubcortical connections that include the thalamus.[26, 34] The proper activation of the precuneus has been considered to be critical in the recovery of low-level states of consciousness and has been considered to be a key player in the default mode network.[35-36] In addition, the precuneus appears to be involved in the management and processing of information from different remote cortical areas, mainly the frontal and parieto-temporal cortices.[37] As expected, our results showed that the functionality of the precuneus differs from that of the other cortical structures, which suggests that functional recovery in this structure tends to progress at a slower rate. The persistence of metabolic changes in the precuneus along the entire clinical spectrum of our sample suggests that this structure may play a central role in the recovery of consciousness [8], which reinforces its role as a cortico-cortical hub. In this sense, the loss of connectivity between the precuneus and the frontal or temporal areas has been associated with autism and cognitive impairment with a high risk of developing Alzheimer`s disease, respectively.[33]

It is now assumed that brain functions, from the basic mechanism that regulates consciousness to the most complex cognitive processes, are not located in specific brain areas of isolated functioning but occur via intricate neural connections underlying the dynamic interactions between brain regions.[38] Our results showed a strong correlation 
in glucose metabolism between all cortical structures, including patients and healthy controls. In cases of severe TBI, the process of the recovery of consciousness and the recovery of the rest of lost skills could be interpreted as a continuum, which only expresses the diffuse loss and the subsequent recovery of brain connectivity. The different metabolic correlations found between patients and healthy controls in terms of thalamo-cortical connections, especially those found in the fronto-thalamic system, suggest that cortico-cortical and cortico-subcortical networks do not have to follow a common pattern of activation and that both systems are not as susceptible to traumatic injury. The weaker correlation detected in the fronto-thalamic system of our healthy controls may be related to the global integrity of the system and its ability to distribute information through a wide network of structurally intact cortico-subcortical connections.[9] Limitations in the sample size or in the percentage of patients with cortical focal lesions found in our study should be addressed in future studies to shed more light on these connections.

In conclusion, our results can help us understand the clinical and neurological evolution of TBI in patients at all levels of dysfunction, from less severe neurological conditions to the most devastating. The gradual activation of the cortico-subcortical systems and the subsequent activation of intercortical connectivity can help determine the most effective treatment in these patients. 


\section{BIBLIOGRAPHY}

1. Gennarelli TA, Graham DI. Traumatic Brain Injury. Part I - Epidemiology and Pathophysiology: Neuropathology. In: Silver JM, McAllister TW, Yudofsky SC, eds. Textbook of Traumatic Brain Injury. Washington D.C. London: American Psychiatric Publishing, Inc.; 2005. p. 27-51.

2. Turner-Stokes L. Evidence for the effectiveness of multi-disciplinary rehabilitation following acquired brain injury: a synthesis of two systematic approaches. J Rehabil Med. 2008;40:691-701.

3. Grados MA, Slomine BS, Gerring JP, et al. Depth of lesion model in children and adolescents with moderate to severe traumatic brain injury: use of SPGR MRI to predict severity and outcome. J Neurol Neurosurg Psychiatr. 2001;70:350-8.

4. Meythaler JM, Peduzzi JD, Eleftheriou E, et al. Current concepts: diffuse axonal injury-associated traumatic brain injury. Arch Phys Med Rehabil. 2001;82:146171.

5. Scheid R, Walther K, Guthke T, et al. Cognitive sequelae of diffuse axonal injury. Arch Neurol. 2006;63:418-24.

6. Bassett DS, Bullmore ET. Human brain networks in health and disease. Curr Opin Neurol. 2009;22:340-7.

7. Sporns O, Honey CJ. Small worlds inside big brains. Proc Natl Acad Sci U S A. 2006;103:19219-20. 
8. Laureys S, Lemaire C, Maquet $\mathrm{P}$, et al. Cerebral metabolism during vegetative state and after recovery to consciousness. J Neurol Neurosurg Psychiatry. 1999;67:121.

9. Mitelman SA, Byne W, Kemether EM, et al. Metabolic thalamocortical correlations during a verbal learning task and their comparison with correlations among regional volumes. Brain Res. 2006;1114:125-37.

10. Zhang D, Snyder AZ, Fox MD, et al. Intrinsic functional relations between human cerebral cortex and thalamus. J Neurophysiol. 2008;100:1740-8.

11. Laureys S, Goldman S, Phillips C, et al. Impaired effective cortical connectivity in vegetative state: preliminary investigation using PET. Neuroimage. 1999;9:377-82.

12. Lull N, Noe E, Lull JJ, et al. Voxel-based statistical analysis of thalamic glucose metabolism in traumatic brain injury: relationship with consciousness and cognition. Brain Inj. 2010;24:1098-107.

13. Kennedy MR, Coelho C, Turkstra L, et al. Intervention for executive functions after traumatic brain injury: a systematic review, meta-analysis and clinical recommendations. Neuropsychol Rehabil. 2008;18:257-99.

14. Levin H, Kraus MF. The frontal lobes and traumatic brain injury. $J$ Neuropsychiatry Clin Neurosci. 1994;6:443-54.

15. Ashburner J, Friston KJ. Voxel-based morphometry--the methods. Neuroimage. 2000;11:805-21. 
16. Good CD, Johnsrude IS, Ashburner J, et al. A voxel-based morphometric study of ageing in 465 normal adult human brains. Neuroimage. 2001;14:21-36.

17. Genovese CR, Lazar NA, Nichols T. Thresholding of statistical maps in functional neuroimaging using the false discovery rate. Neuroimage. 2002;15:870-8.

18. Boly M, Phillips C, Tshibanda L, et al. Intrinsic brain activity in altered states of consciousness: how conscious is the default mode of brain function? Ann N Y Acad Sci. 2008;1129:119-29.

19. Hattori N, Huang SC, Wu HM, et al. Correlation of regional metabolic rates of glucose with glasgow coma scale after traumatic brain injury. $J$ Nucl Med. 2003;44:1709-16.

20. Kato T, Nakayama N, Yasokawa Y, et al. Statistical image analysis of cerebral glucose metabolism in patients with cognitive impairment following diffuse traumatic brain injury. J Neurotrauma. 2007;24:919-26.

21. Nakashima T, Nakayama N, Miwa K, et al. Focal brain glucose hypometabolism in patients with neuropsychologic deficits after diffuse axonal injury. AJNR Am J Neuroradiol. 2007;28:236-42.

22. Nakayama N, Okumura A, Shinoda J, et al. Relationship between regional cerebral metabolism and consciousness disturbance in traumatic diffuse brain injury without large focal lesions: an FDG-PET study with statistical parametric mapping analysis. J Neurol Neurosurg Psychiatry. 2006;77:856-62. 
23. Wu HM, Huang SC, Hattori N, et al. Selective metabolic reduction in gray matter acutely following human traumatic brain injury. J Neurotrauma. 2004;21:14961.

24. Laureys S, Owen AM, Schiff ND. Brain function in coma, vegetative state, and related disorders. Lancet neurology. 2004;3:537-46.

25. Schiff ND, Ribary U, Moreno DR, et al. Residual cerebral activity and behavioural fragments can remain in the persistently vegetative brain. Brain. 2002;125:1210-34.

26. Laureys S, Faymonville ME, Luxen A, et al. Restoration of thalamocortical connectivity after recovery from persistent vegetative state. Lancet. 2000;355:1790-1.

27. Anderson CV, Wood DM, Bigler ED, et al. Lesion volume, injury severity, and thalamic integrity following head injury. J Neurotrauma. 1996;13:35-40.

28. Fernandez-Espejo D, Junque C, Bernabeu M, et al. Reductions of thalamic volume and regional shape changes in the vegetative and the minimally conscious States. J Neurotrauma. 2010;27:1187-93.

29. Nakayama N, Okumura A, Shinoda J, et al. Evidence for white matter disruption in traumatic brain injury without macroscopic lesions. J Neurol Neurosurg Psychiatry. 2006;77:850-5. 
30. Wilson JT, Hadley DM, Wiedmann KD, et al. Neuropsychological consequences of two patterns of brain damage shown by MRI in survivors of severe head injury. J Neurol Neurosurg Psychiatry. 1995;59:328-31.

31. Wilson JT, Teasdale GM, Hadley DM, et al. Post-traumatic amnesia: still a valuable yardstick. J Neurol Neurosurg Psychiatry. 1994;57:198-201.

32. Tate RL, Pfaff A, Jurjevic L. Resolution of disorientation and amnesia during post-traumatic amnesia. J Neurol Neurosurg Psychiatry. 2000;68:178-85.

33. Tullberg M, Fletcher E, DeCarli C, et al. White matter lesions impair frontal lobe function regardless of their location. Neurology. 2004;63:246-53.

34. Cavanna AE. The precuneus and consciousness. CNS Spectr. 2007;12:545-52.

35. Cruse D, Owen AM. Consciousness revealed: new insights into the vegetative and minimally conscious states. Curr Opin Neurol. 2010;23:656-60.

36. Fransson P, Marrelec G. The precuneus/posterior cingulate cortex plays a pivotal role in the default mode network: Evidence from a partial correlation network analysis. Neuroimage. 2008;42:1178-84.

37. Cavanna AE, Trimble MR. The precuneus: a review of its functional anatomy and behavioural correlates. Brain. 2006;129:564-83. 
38. Fuster JM. The cognit: a network model of cortical representation. Int $J$ Psychophysiol. 2006;60:125-32. 


\section{Legend Figure 1}

Figure 1. Axial and sagital (right) metabolic differences (Z-values) between the controls and the patients. Upper part: metabolic comparison between the controls and the MCS\&VS group showing differences in all four structures. Middle part: metabolic comparison between the controls and the In-PTA group showing differences in all four structures. Lower part: metabolic comparison between the controls and the Out-PTA group showing differences in the thalamus and the frontal cortex. As the intensity scale shows, brighter areas indicate the greater differences.

\section{Legend Figure 2}

Figure 2. Axial and sagital (right) metabolic differences (Z-values) between the patients. Upper part: metabolic comparison between the Out-PTA group and the MCS\&VS group showing differences in the thalamus, the frontal and temporal cortex. Middle part: metabolic comparison between the Out-PTA group and the In-PTA group showing differences in all four structures. Lower part: metabolic comparison between the In-PTA group and the MCS\&VS group showing differences in the thalamus. As the intensity scale shows, brighter areas indicate the greater differences.

\section{Legend Figure 3}

Figure 3. Correlation analysis: $\mathrm{X}$ axis represents patients and $\mathrm{Y}$ axis shows contrast intensity values. 


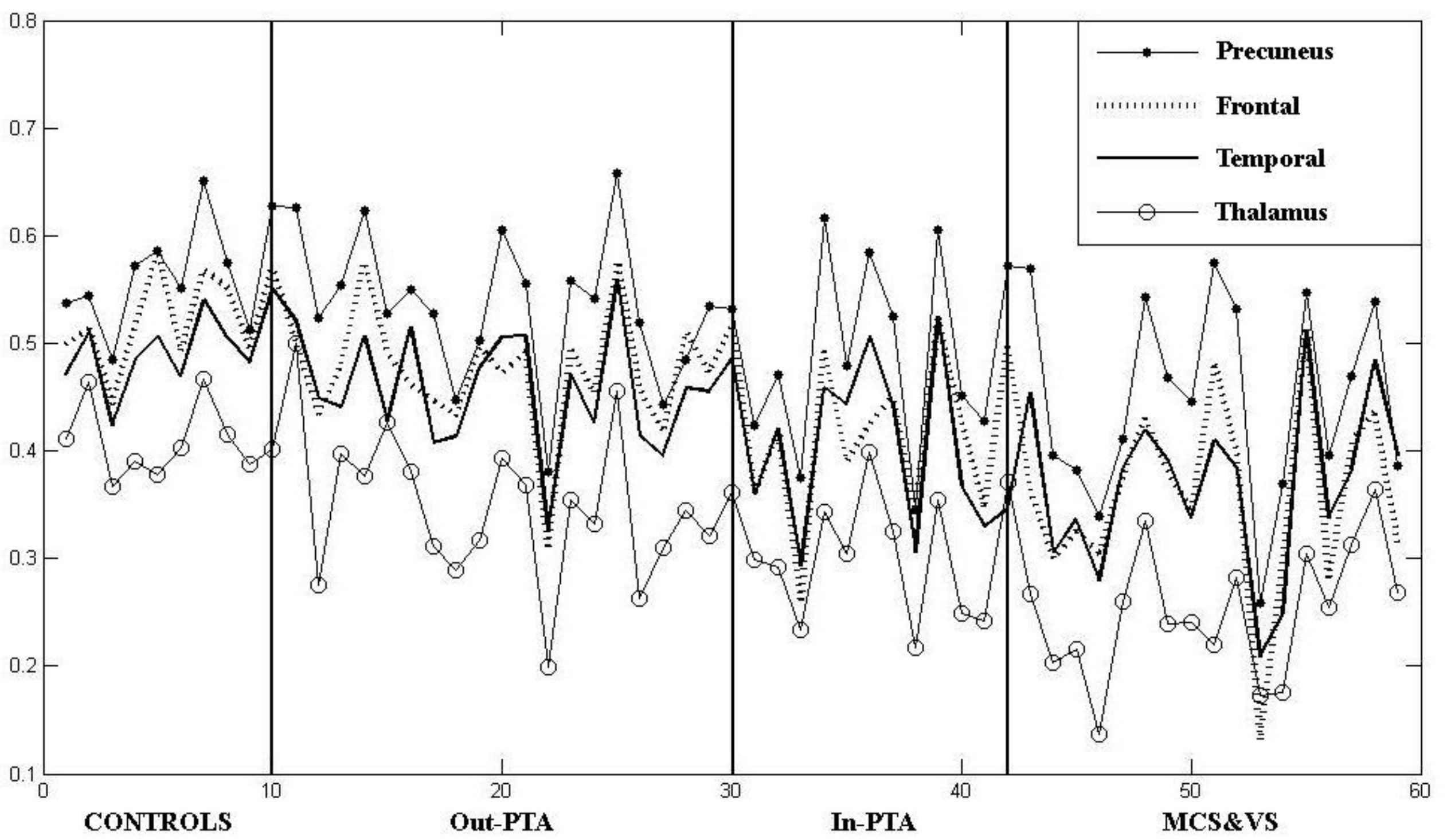

